\title{
Web-based Remote Control System for IPTV Service
}

\author{
Kangwon Lee, Yejin Shin, Jihyun Jo, Jinseo Jeong, Seongho Choo, Yeonji Lee, \\ and Soonuk Seol* \\ School of Electrical, Electronics and Communication Engineering \\ Korea University of Technology and Education \\ Cheonan, South Korea \\ \{kangwon1020, yepp1252, hg5312, walend, csh9895, goooodgood, \\ suseol\}@koreatech.ac.kr
}

\begin{abstract}
Recently IPTV service has been widely deployed, but the disabled and the elderly are not easy to fully utilize various features of IPTV service. To resolve this problem, in this paper, we propose a web-based remote control approach where a remote user can access the IPTV system and manipulate it. In order to show feasibility, we actually implement an IPTV set-top software which employs our software libraries and develop a Web server. An experimental result shows that our proposed system can be effectively utilized for the disabled with a very low data usage and a reasonable response time.
\end{abstract}

Keywords: Remote Control, IPTV, IoT, HCI, Smartphone, Web, Disabled

\section{Introduction}

The development of new technology has led to the emergence of Internet Protocol Television (IPTV), which provides various services including video on demand for TV dramas and movies, web surfing, shopping, and games, in contrast to conventional TVs which only allow for real-time viewing. While the duration of TV utilization is increased as consumers utilize TVs in various ways, the TV utilization level is different among different age groups and social groups. According to a survey performed by the Korea Information Society Development Institute in 2015, the dependence on TV for leisure was $19.0 \%$ among adults in their $20 \mathrm{~s}$, but $46.2 \%$ among adults in their $60 \mathrm{~s}$ or higher. In addition, 'Investigation of Current Status of Disabled Persons 2011' conducted by the Ministry of Health and Welfare showed that TV watching accounted for $96 \%$ of the leisure activities by the disabled. Although TV watching accounts for the largest share of leisure activities by the disabled and the elderly, whether they sufficiently utilize multifunctional IPTV is still doubtful. One survey showed that the rate of using the VOD service was as low as 2.2 among TV viewers aged 60 years or higher, indicating that they have a passive TV watching pattern of watching real-time broadcast programs rather than actively choosing desired contents.

Unlike conventional TVs which could be controlled by channel shifting and volume control only, the input methods, such as contents search and payment, are diverse, and the control process is complicated in IPTVs. For example, access to the VOD service requires many inputs on the menu by using the remote controller. As such, the elderly and the disabled have difficulty accessing the IPTV services.

Various studies have been conducted to aid in IPTV control by the elderly and the disabled. However, most of the previous studies have focused on improvement of input methods through motion recognition in the aspect of the interaction between a user and TV [1-2], rather than improving the recognition of the complex TV menus and the choice of appropriate items. As such, the findings of previous studies may not provide practical

* Corresponding author: Soonuk Seol, Email:suseol@koreatech.ac.kr 
help to people who lack an understanding of the constitution and functions of IT devices or who have difficulties in handling unexpected results of TV manipulation.

To overcome these shortcomings, a web-based IPTV remote control system was designed and realized in this paper. A person acting on behalf of a disabled person may use the system proposed in our proposed system to help to play the desired content. This may increase media accessibility for the disabled and IT-alienated, as well as the level of IT device utilization.

This paper includes the following chapters. Chapter 2 describes conventional approaches to TV control methods, and introduces the remote control platforms proposed in previous studies and their applications. Chapter 3 describes the improvement of the authors' platform for remote control of IPTV. Chapter 4 introduces the results of the realization of the IPTV remote control system. Finally, Chapter 5 presents the conclusions of the proposed system and future studies.

\section{Related Work}

\subsection{Control Method}

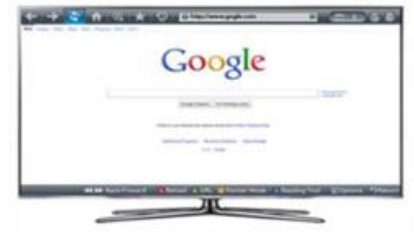

Current Tab

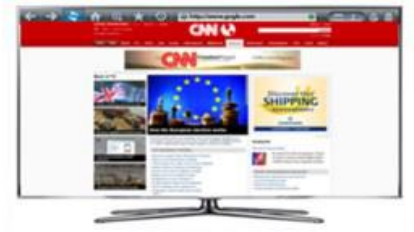

Next Tab

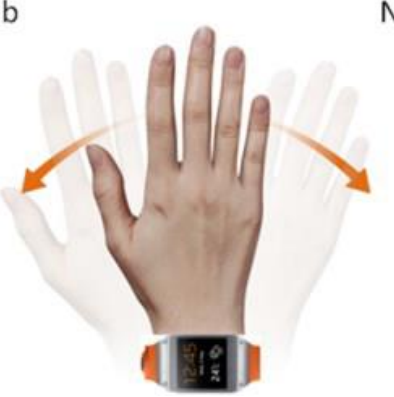

Gesture to switch tab (recognized using wearable device or mobile)

\section{Figure 1. Illustration of the Web Browser on TV with Two Different Browser Tabs}

The first approach is a method of controlling the TV through motion recognition. This method assists in intuitive TV control based on user interface improvement. Seetharamu [1] developed a TV control method using a smart watch, wherein a user's motion is sensed through a sensor embedded in a smart watch and the sensed data are transmitted to a smartphone which then controls the web browser on the TV as shown in Figure 1. The smartphone determines the kinds of motion made by the user on the basis of the received data, and then transmits the control operation corresponding to the user's motion to the TV. Similarly, Keita Watanabe studied a method of controlling a TV by recognizing a user's motion through a Kinect separately installed beside a TV [2]. This method sets the coordinates of basepoint to the center of both shoulders and it also sets the coordinates of operating point to the hand. Then, the intersection point of the screen and the extension line made from the two points is defined as a coordinates of a pointer like a mouse cursor as shown in Figure 2. 


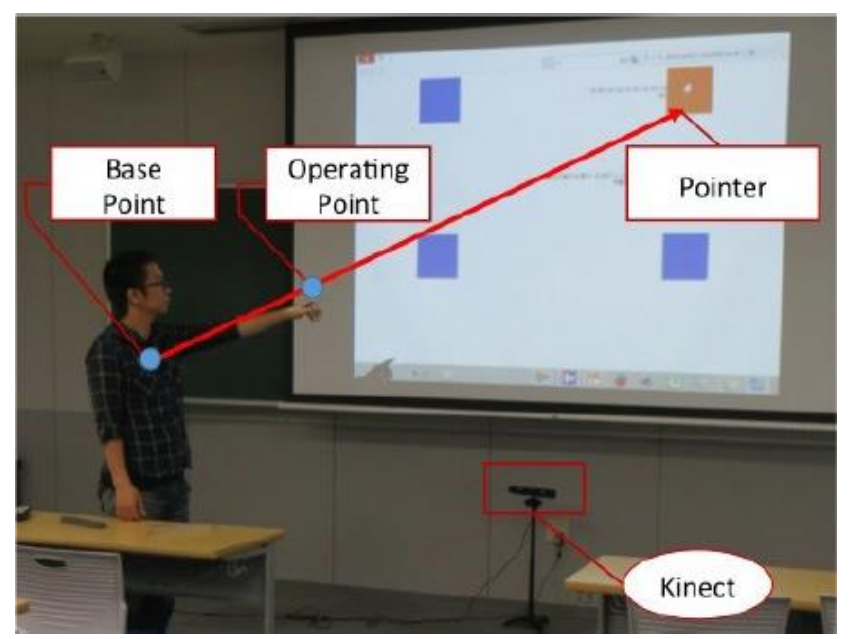

Figure 2. Example of Using Remote Touch Pointing

Another approach is a touch-based TV control method using a smartphone application that plays the role of a TV remote controller [6, 7]. However, these approaches may not provide practical help to people who lack an understanding of the constitution and functions of IT devices, or who have difficulties in handling the unexpected results of TV manipulation.

On the other hand, products such as Remote Desktop [8] and TeamViewer [9] are also available, and these enable the sharing and control of a computer screen based on streaming, but these products are difficult to apply to IT devices other than computers. In particular, the embedded system with limited resources may have insufficient processing performance. For embedded IT devices, our previous study $[3,4,5]$ takes a car navigation system as a remote control example. In these work, user's smartphone is used as a mobile gateway for those devices that have no mobile communication function. The menu information has been reconstructed in the form of text-based information to minimize data consumption, allowing for control through a web browser. However, services with graphics-based menus, including movie posters on IPTV, have limitations when it comes to applying them to a text-based menu. In this paper, a web-based remote IPTV control system was designed and realized that allows for transmission of a captured image of a local screen when needed for convenient control.

\subsection{Web-based Remote Control Platform}

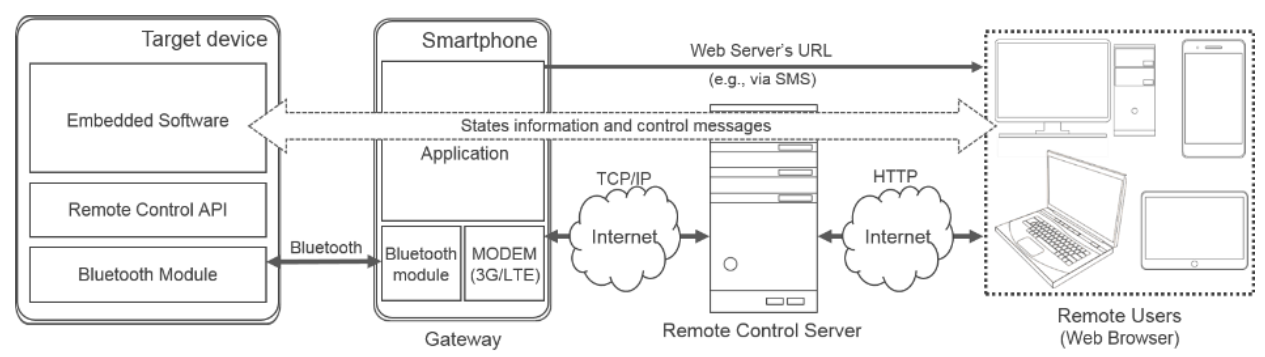

Figure 3. Architecture of Remote Control Platform

In our previous study, we proposed a web-based remote control platform to provide easy access to various devices. As shown in Figure 3, the proposed platform consists of a target device, a user's smartphone, a mediating server, and a controller's smartphone (or a laptop computer, tablet PC, etc.). The target device includes a Bluetooth module, and exchanges state information and control information of the target device with a user's 
smartphone through Bluetooth communication. The smartphone exchanges information about the target device through communications technologies such as 3G, LTE, and Wi-Fi. The target device may be directly connected to the server. However, an off-line device that may not be directly connected to the internet may be connected to the Internet by using a user's smartphone as a gateway.

A Node.js-based mediating server communicates with the smartphone, and creates a web page about the received information regarding the state of the target device, providing a URL that allows the controller to access the created web page. The controller may access the web page by using a smartphone or PC to view the state information of the target device and control the device.

\subsection{Limitations of Conventional Platforms}

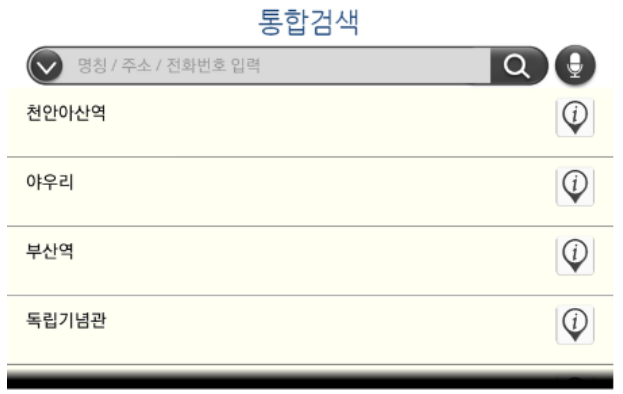

(a)

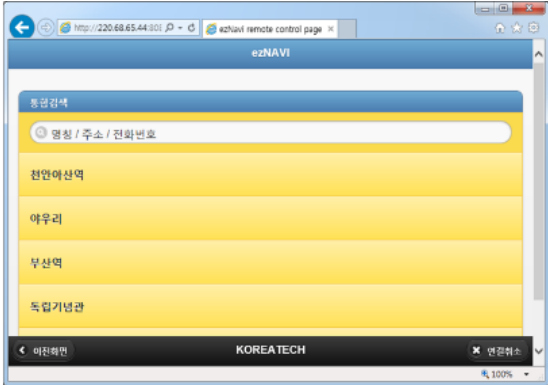

(b)

\section{Figure 4. Comparison of the Target Device's Screen and a Remote User's Screen: (a) Car Navigation System, (b) Remote User's Screen}

In our previous study, we applied our remote control platform to a vehicle navigation system $[3,4,5]$. Users can easily perform remote control of a navigation system even if the screen information is shared in the form of text, because the information exchanged is usually simple, such as menu selection and destination search. Therefore, the screen information was transmitted as text in order to create a web page using the text information, as shown in Figure 4. Figure 4(a) shows an actual navigation screen, and 4(b) shows navigation screen information displayed on the created web page accessed through a controller's web browser.

However, as shown in Figure 5, a great deal of information is displayed on an IPTV screen because the menu configuration is complex, and includes a main menu, sub-menus, and shortcut menus. As such, a controller may not easily understand the TV's state if the screen information is transmitted in the form of text. In addition, the VOD contents provided by IPTV may be chosen with reference not to the title of the contents but to the relevant images, such as movie posters. Since the service provided by IPTV is basically an image-based service, the user's range of choice may be varied depending on the presence of images. Therefore, remote control of IPTV may be limited if the screen information is transmitted only in the form of text.

Applying a streaming-based control method to overcome the problems will bring about an increase of data consumption due to the real-time image transmission of IPTV. In addition, the operation may not be smooth in the set-top box, considering that it has a lower level of hardware performance in comparison with PCs and smartphones. To resolve these problems, the system proposed in this paper captures and transmits a still image by sensing the IPTV screen change. A remote controller may obtain enough information to control the IPTV with little data consumption, and control the menu more intuitively in comparison with menus shown in text. 


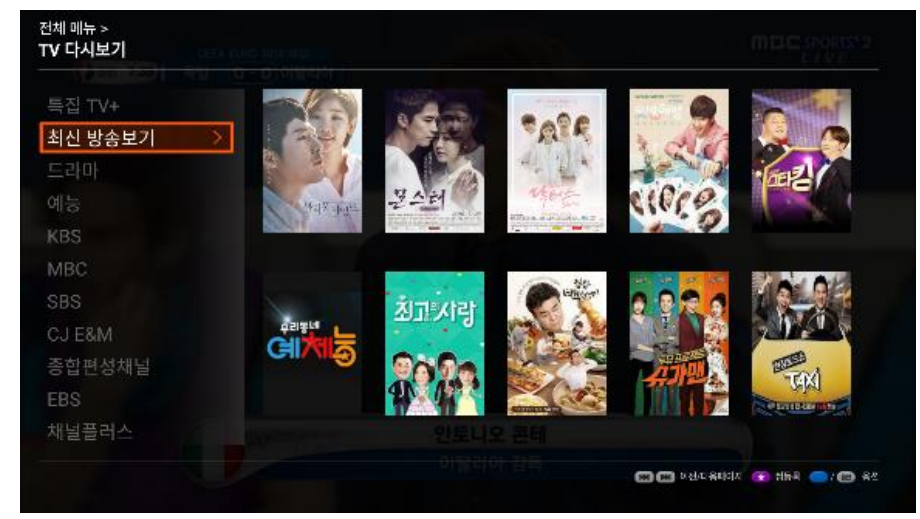

Figure 5. VOD Menu Screen

\section{IPTV Remote Control System Design}

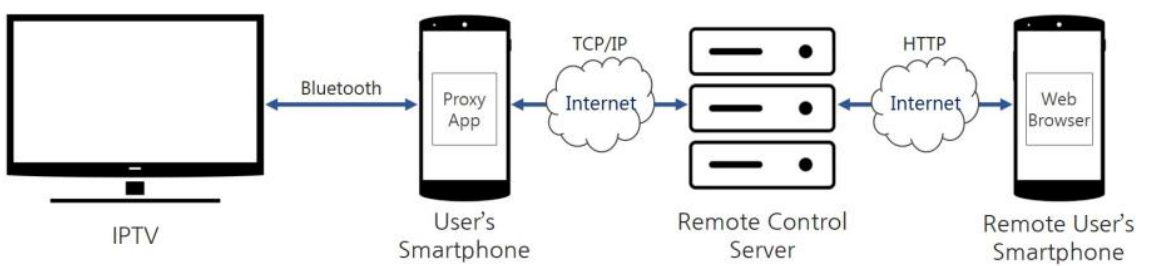

Figure 6. IPTV Remote Control System Architecture

The remote control system is improved to apply the platform proposed in our previous study to IPTV. As shown in Figure 6, the designed system consists of the IPTV, a user's smartphone, a mediating server, and a controller's smartphone.

\subsection{IPTV}

IPTV is a remotely controllable target device and transmits for control the current state and event information in connection with a proxy app. Figure 7 shows the message format for the transmission of the state and event information.

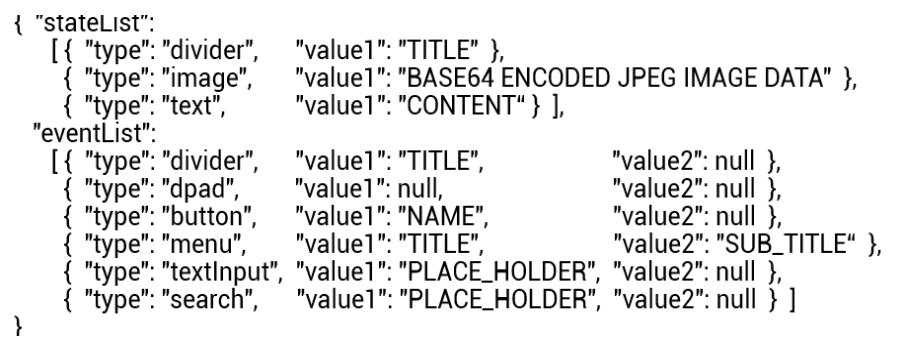

Figure 7. Message Format

The message is divided into the stateList and the eventList, where the stateList includes the state information, such as IPTV screen capture image, channel, and volume. The eventList includes the information about the events that are available on the current screen, such as menu selection button. These lists are converted into JavaScript Object Notation (JSON) format, transmitted to a proxy app, and through the server constitute a control page for a remote user. When the remote controller performs control through the web page, the IPTV control information is transmitted again through the proxy app, and the state of the IPTV is changed according to the control information. When the IPTV screen 
is turned, the new screen capture image and the events available on the screen are transmitted to the server.

\subsection{Proxy}

A proxy app is installed on a smartphone and used to connect the IPTV and the server and mediate the messages through communication. When a user presses the "Connect" button after selecting a controller through the app, the proxy app connects the IPTV through Bluetooth communication. After the Bluetooth connection is completed, the proxy app receives the message including the state and event information from the IPTV. Then, the proxy app connects the mediating server through TCP socket communication. When the connection is completed, the message is transmitted to the server. In addition, the proxy app receives from the server the URL of the web page that the controller may access, and transmits the URL to the smartphone of the controller through SMS. The URL used for the control includes a random code that is valid only for the current session and as such the URL may not be reused by another user or after the completion of the session.

\subsection{Remote Control Web Page}

The mediating server established on the basis of Node.js performs parsing of the state and event information message received from the proxy app to create a web page. The top of the web page accessed by the controller includes a captured IPTV screen. The web page also provides events that are controllable on the screen in the forms of button, list view, D-pad, and Input field. The graphic user interface constituting the web page is a general-purpose UI that is applicable not only to IPTV but also to various other devices including a car navigation device.

The controller may view the state of the IPTV through the web page and control the IPTV using the input buttons under the capture image. When the controller presses one of the buttons, the button information is transmitted to the IPTV, which performs the control operation according to the selected button. When the state and event message about the changed IPTV screen is received, the web page is automatically refreshed. When the control is finished, the communication may be discontinued by pressing the Exit button.

\subsection{Procedure}

Figure 8 shows the procedure of the remote control system for IPTV service. The procedure mainly consists of four parts (A) connection establishment, (B) creation of an initial web page, (C) remote control, and (D) connection close.

\section{A. Connection establishment}

A user chooses a controller and a device such as IPTV, and requests connection through a proxy app. The proxy app is connected by Bluetooth connection with the device. The proxy app is also connected by TCP socket connection with the remote control server.

\section{B. Creation of an initial web page and remote user's access}

The device creates its current state and event information and sends it to the proxy app. The proxy app forwards it to the remote control server so that the server can create an initial web page. The server creates an access URL for the web page and sends it to the proxy app. Then, the proxy app forwards the URL to the controller's smartphone via SMS message. The controller can access the web page by using the URL in the SMS message.

\section{Remote control}

The controller clicks a menu or enter a keyword in the remote control web page. Then, a control message is delivered to the remote control server. The server forwards the 
message to the device via the proxy app. Upon receiving the control message, the device will operate accordingly. The updated state and event information is sent to the remote control server. If the server receives new information, it refreshes the web page by using the received information. Then, the controller can make the subsequent controls by doing the above process repeatedly

\section{Connection close}

If the controller clicks a 'Exit' button in the web page, the exit message is sent to the proxy app via the remote control server. When the proxy app receives the exit message, the proxy app requests connection close to the device and remote control server, respectively. And then, the Bluetooth socket with the device and TCP socket with remote control server are closed.

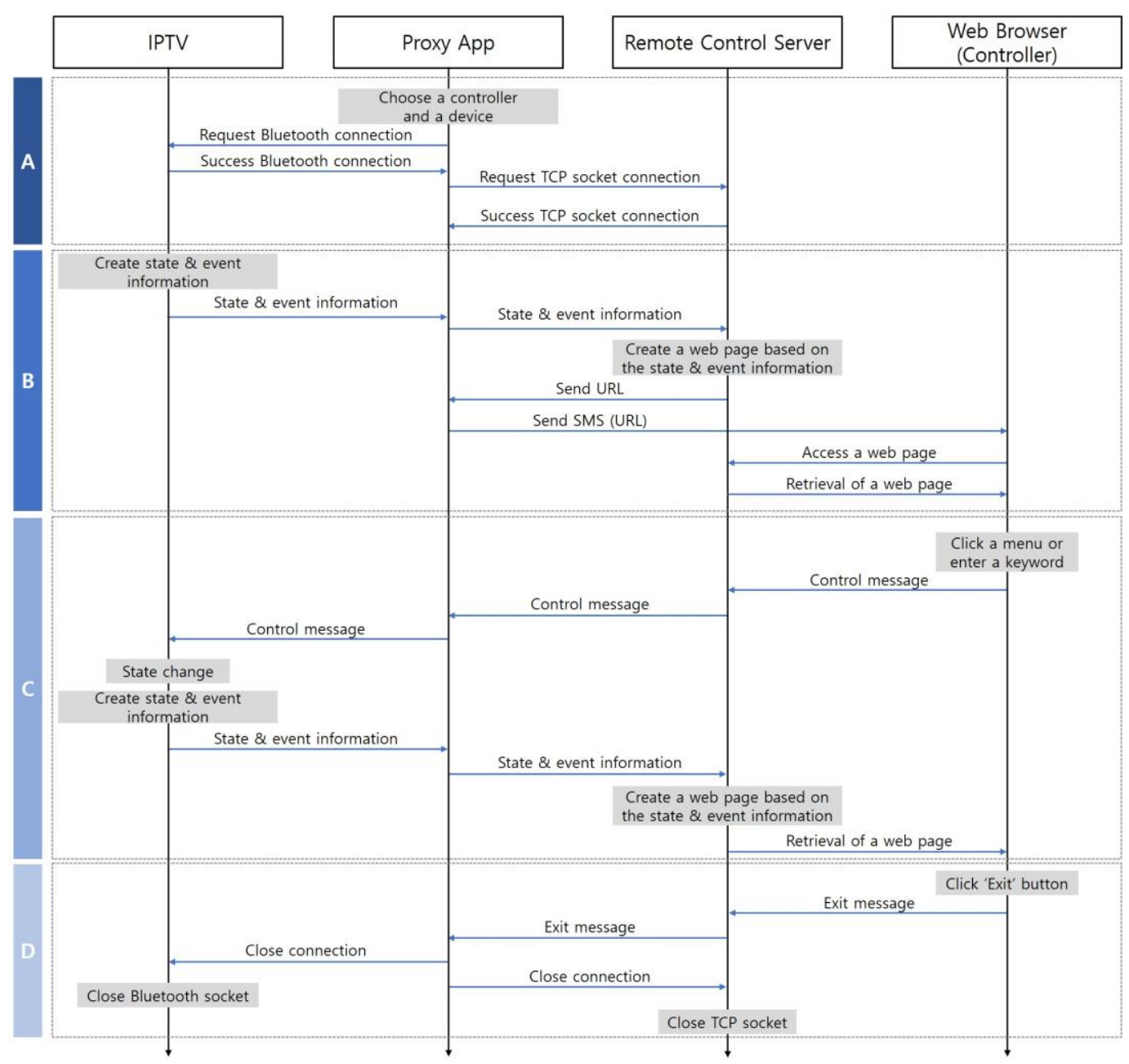

Figure 8. The Procedure of Remote Control System 


\section{Realization Result}

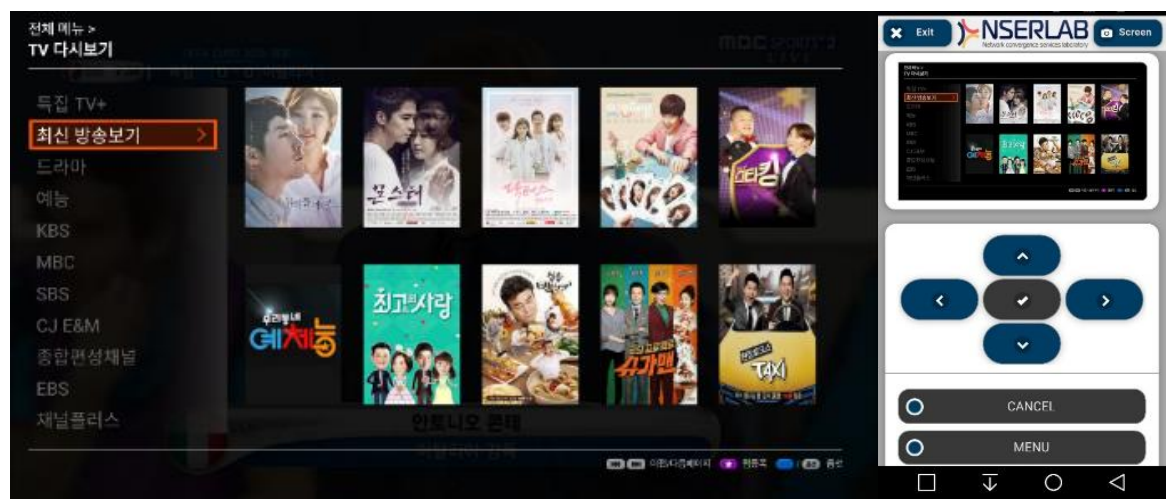

Figure 9. Demonstration Screen

To demonstrate the proposed system, an IPTV was developed as an Android-based TV app, which was then installed on the NVIDIA SHIELD Android TV, a smart set-top box. A proxy app for the connection between the TV app and the server and the message mediation was installed on an LG G5 smartphone, and an LG G4 smartphone was used as the controller's terminal. Figure 9(a) shows the screen of the executed IPTV developed as an Android-based app, and 9(b) shows the accessed web page created according to the transmitted current screen and event information from the IPTV.

The realized system enables more intuitive control of the IPTV in comparison with the previous text-based navigation platform, because the system of the proposed system shows the captured image of the IPTV screen. In addition, the captured image is transmitted only when the screen is changed by control, and thus the data consumption for each page is just about 14 kilobytes. Thus, the proposed system may reduce data consumption in comparison with streaming-based PC and smartphone remote control. The round trip time (RTT) from the time when a remote control action was performed by the controller through the web page of the system to the time when the information about the next screen was transmitted was about 0.88 seconds. This means that the utilization may be convenient.

\section{Conclusions and Future Study}

In this paper, a system that enables a remote controller to control an IPTV on behalf of a user was proposed in order to liberate the disabled or the elderly from complex remote controller manipulation to help them to use the various functions of IPTV. The system was designed and realized for web-based remote control of IPTV. We verified that the realized system enabled intuitive IPTV control on the basis of image-based information at an acceptable response time, using small data consumption.

The proposed system may help the disabled or the elderly alone at home to be liberated from the complex TV control and to utilize various IPTV contents by asking an acquaintance to perform IPTV control on their behalf. In addition, it may be applied to help the disabled or the elderly to utilize various other things that they could not easily use, contributing to the advancement of human care IT for an information-alienated sector of society.

\section{Acknowledgments}

This work was supported by the National Research Foundation of Korea(NRF) grant funded by the Korea government(MSIP) (No. NRF-2015R1A2A1A16074941). 


\section{References}

[1] V. K. Seetharamu, J. Bose, S. Sunkara and N. Tigga, "TV Remote Control via Wearable Smart Watch Device", Annual IEEE India Conference, (2014), pp. 1-6.

[2] K. Watanabe, Y. Miyake, N. Nakamichi, T. Yamada and T. Ozeki, "Remote Touch Pointingfor Smart TV Interaction”, IEEE ${ }^{\text {rd }}$ Global Conference on Consumer Electronics, (2014), pp. 232-235.

[3] Y. Shin and S. Seol, "Design and Implementation of Remote Control System for Car Navigation", The Journal of the Korea Institute of Information and Communication Engineering (KIICE), vol. 18, no. 7, (2014), pp. 1695-1703.

[4] Y. Shin and S. Seol, "Smartphone as a Remote Control Proxy in Automotive Navigation System", Contemporary Engineering Sciences, vol. 7, no. 14, (2014), pp. 683-689.

[5] Y. Shin, S. Seol and K. Lee, "A Study on Quality of Experience of Controlling a Device Remotely in an IoT Environment", 2016 Eighth International Conference on Ubiquitous and Future Networks, (2016), pp. 699-702

[6] H. H. Chen, Y. B. Lin, Y. C. Sung and R. H. Liou, "Direction-based Wireless Remote Controller: A Smartphone Application", Journal of Wireless Mobile Networks, Ubiquitous Computing, and Dependable Applications., vol. 2, no. 2, (2011), pp. 33-45.

[7] T. F. Chueh and Y. Y. Fanjiang, "Universal remote control on smartphone", Computer, Consumer and Control (IS3C), International Symposium on. IEEE, (2012), pp. 658-661.

[8] Remote Desktop, www.microsoft.com

[9] TeamViewer, www.teamviewer.com
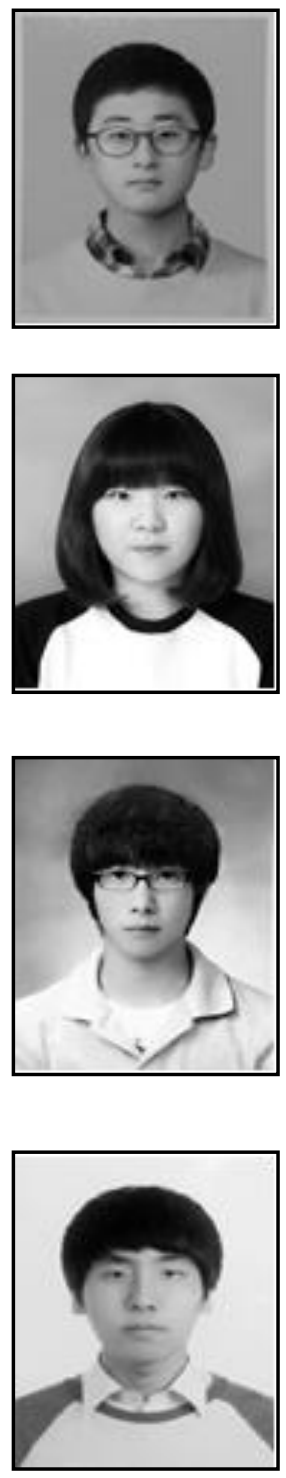

\section{Authors}

Kangwon Lee, he received the B.S. degree from Korea University of Technology and Education (KOREATECH) in 2015. He is pursuing the M.S. degree in Information and Communication Engineering from KOREATECH. He is interested in IoT, QoS, mobile internet, etc.

Yejin Shin, she received the B.S. and M.S degree from Korea University of Technology and Education (KOREATECH) in 2014 and 2016. She is pursuing the Ph.D. degree in Information and Communication Engineering from KOREATECH. She is interested in wireless telecommunication system, wireless LAN, RFID/USN, etc.

Jihyun Jo, he is pursuing the B.S. degree in Information and Communication Engineering from KOREATECH. He is interested in IoT, wireless LAN, SDN/NFV, etc.

Jinseo Jeong, he is pursuing the B.S. degree in Information and Communication Engineering from KOREATECH. He is interested in mobile internet, IoT, cloud computing, etc. 

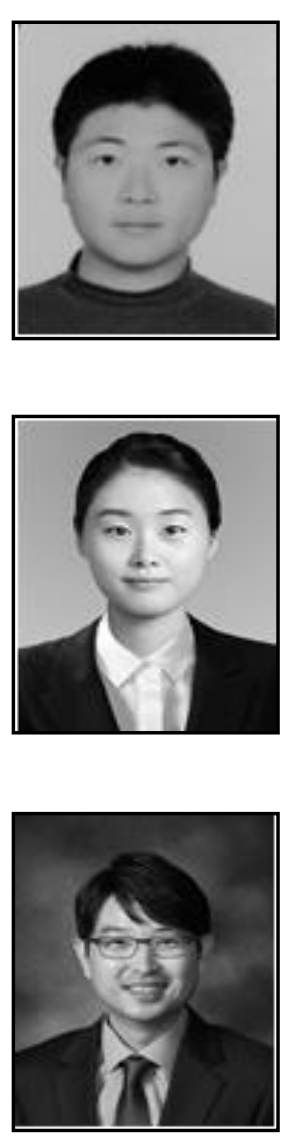

Seongho Choo, he is pursuing the B.S. degree in Information and Communication Engineering from KOREATECH. He is interested in mobile internet, IoT protocols, wireless LAN, etc.
Yeonji Lee, she received the B.S. degree from Korea University of Technology and Education (KOREATECH) in 2016. She is pursuing the M.S. degree in Information and Communication Engineering from KOREATECH. She is interested in wireless LAN, SDN/NFV, multi-hop ad hoc networks, etc.

Soonuk Seol, he received his B.S. degree from Korea University of Technology and Education (KOREATECH) in 1998, M.S and $\mathrm{Ph}$.D. degrees in Information and Communication Engineering from KAIST in 2000 and 2004, respectively. He worked as a senior researcher at KT from 2004 to 2012 . He is currently an associate professor in School of Electrical, Electronics, and Communication Engineering at KOREATECH. His research interests include mobile Internet, IoT, QoS, and software testing. 